\title{
Chapter 9 \\ Between Presence and Program: The Photographic Error as Counter Culture
}

\author{
Tracy Piper-Wright
}

\begin{abstract}
Common photographic errors such as over or under exposure, blur, or inadvertent cropping are increasingly rare as technological developments in digital photography have sought to eradicate the error from practice and perception. Efficiencies such as camera automation and image preview are often designed to remove the 'unreliability' of the human element in order to produce accurate and consistent images. The error, occurring on the margins of practice and increasingly rare, provides a counterpoint to this prevailing image culture by revealing the interdependence of photographer and camera through unintended outcomes. This chapter explores the ideological assumptions entwined in the development of camera technologies, and how cultures of practice based on a hierarchy of control between camera and photographer arose. Through examples drawn from the research project In Pursuit of Error, the chapter demonstrates how the error disrupts this hierarchy by evidencing the shared subjective agency of camera and photographer. The methodological framework of Actor-Network Theory is used to interrogate the relationship between photographer and camera and to reveal them as equal 'actants' in the event of photographing. The embodied photographer's attitude of play, experimentation, and not-knowing is interdependent on the camera as a co-creator of unexpected image events which disrupt the conventions of photographic representation.
\end{abstract}

Keywords Photography $\cdot$ Error $\cdot$ Agency $\cdot$ Doubt $\cdot$ Actor-Network $\cdot$ Embodiment $\cdot$ Subjectivity $\cdot$ Time $\cdot$ Hierarchy of agency $\cdot$ Digitization $\cdot$ Virtuality $\cdot$ Actor-Network Theory $\cdot$ Transformative art $\cdot$ Alternative realities Presence $\cdot$ Cultures of practice $\cdot$ Experimental photography

\subsection{Introduction: Histories and Hierarchies}

Alone of all the creative arts, photography is the most deeply enmeshed with the technologies of production. A photograph cannot be made without an instrument external to the photographer whereas one might argue the technologies of drawing

\footnotetext{
T. Piper-Wright $(\varangle)$

University of Chester, Chester, UK

e-mail: t.piperwright@ chester.ac.uk

(C) The Author(s) 2020

R. Earnshaw et al. (eds.), Technology, Design and the Arts-Opportunities and Challenges, Springer Series on Cultural Computing, https://doi.org/10.1007/978-3-030-42097-0_9
} 
or painting can, on occasion, be replaced by the human hand and the right surface. The symbiosis between camera and photographer makes for a peculiar push and pull between the claims of the technical and the subjective, which have been the hallmark of writing about photography as, variously, an art, a craft and a science since the inception of the medium. The tension between an apparently objective and automatic picturing mechanism and the role of the photographer in the creation of the image dates back to the critics and practitioners of the late nineteenth century, and centers around a hierarchy of agency — who or what is finally responsible for the photograph?

The unavoidable connectedness between camera and photographer is not a fault of the practice, but rather an important distinction which must be recognized in any discussions about it. In the early twenty-first century the digitization of photography coincided with the connectivity offered by the Internet, producing the circumstances for limitless digital images to be created and subsequently shared and viewed in distributed networks. This rapid development created extra layers of technological distance between photographer and camera as points of tension emerged between the physicality of analog film and the 'virtuality' of the digital photograph [1, p. 97]. As digital cameras become increasingly abstruse and proliferate the question of the relationship between it and the photographer becomes one of knowledge as well as agency. If I cannot know what the camera is actually doing, how much control can I have? The "black box" [2, p. 27] of the digital camera lies so far outside the realms of ordinary understanding that photography has almost returned to the realm of the magical.

What made photography 'magical', in its earliest presentations, was its ability to capture and retain an image of the world with a precision hitherto impossible through the human hand alone [3]. While early discussions on the invention of photography refer to the photographic process rather than the camera itself; the technology of the camera as an anthropomorphized 'eye' was first proposed by Da Vinci in relation to the camera obscura [4] the earliest form of enclosed light projection from which cameras were developed. Thus, some assumptions about photography were set out at the early stage of its development: a monocular, perspectival viewpoint which mimics the focus of attention in human vision and a unique responsibility to reality which is the product of an "unreasoning machine" that produces "an unerring record" [5, p. 269].

The camera's 'eye' supersedes the frailties of the human eye through its objective stance and ability to transmit and reveal the world as it is, without interpretation or editing. The camera becomes a technology for asserting truth, and the images it produces are facts that can be used as evidence.

Leading from its perceived status as a truth-telling machine, the quest for simplified and accessible accuracy has been a dominant motivator in the technological development of the camera. The famous Kodak strap line which accompanied the launch of its camera in 1888 "You push the button - we do the rest" [6, p. 38] exemplified a desire to remove the possibility of error in the creation of a photograph by eliminating the more complicated or technical aspects both in camera design (the camera had a fixed focus lens and limited aperture/shutter speed controls) and in 
the loading and development of film (the whole camera was sent back to Kodak for processing, thence replenished and returned to the owner) [6].

The Kodak camera is evidence of the twin aims of technological progress which can be found in most subsequent camera development including digital. Firstly, there is the desire to ameliorate the complexity of the photographic process per se; the unknowable chemical/algorithmic functions which are performed in the making of the image. Secondly, the camera sets out to moderate the potential of a faulty, errorprone human element from entering the photographic equation. The photographer is reduced to being a button pusher, and no more. The twin aims of camera development: simplicity and error elimination can be seen in many of the innovations in camera technology in the twentieth century, such as cassette film and auto-focus, which continued into the twenty-first century digital revolution.

These reflections on the early history of photography show that the pursuit of accuracy has been at the forefront of the practice and perception of photography since the beginning. The linking of photography as a technical yet simplified image making method that requires the presence of a 'button pusher' but which devolves the image making to the camera, makes for a peculiar awkwardness in the relationship between human and camera: an oddly mismatched hierarchy in which the photographer is perceived as both necessary and extraneous to the photographic event.

Despite these advances, errors still crept into the practice of photography. Not every eventuality could be eliminated, and the material agency of the film and the chemical process contained many potential pitfalls for the photographer, which were often only revealed once the prints had returned from the developer. Unsatisfactory images could be discarded, but were often simply incorporated back into the wallet of photographs. The gap between the making and revealing of an error effected a nostalgic distance between event and object in which the significance of the image could override the obvious flaws in the depiction. In the analog era photographs were perceived as a finite resource, and an error might appear a charming reminder of a moment in time.

Digital photography took its cues from film photography in terms of camera design and it is therefore unsurprising that its subsequent development extended the simplicity/error-elimination methodology. However, digital photography offered a level of certainty that far exceeded what had been possible before. Due to the algorithmic nature of the sensor and digital processor cameras became computers, and the photographer became less and less the primary decision-maker [7]. Later developments such as image preview effectively capped the potential for error by making it possible to delete substandard images at source, narrowing the possible images that could emerge from any given shoot. In the digital era the opportunities for 'casual' error creation changed; no longer could a substandard image slip through the net and into the pile of prints. Each image could be vetted so that only the best were eventually presented in online spaces.

As our consumption of photography as digital images in networked environments increases the more our perception of photography reflects what we are presented with-invariably 'good' photographs. In this case a 'good' photograph does all that a photograph should: it shows things as they are, to the best of the camera's ability. 
The technology of the camera, now most often embedded in a phone, can control lighting, saturation, and shadows to produce high quality images that feel 'just like being there'. This quest for transparency, for a feeling that you are looking at or experiencing the 'real thing', has been the ultimate goal of consumer digital photography, with increased mega-pixels offering more and better rendering of the scene. The photograph becomes a window through which we observe the phenomenon depicted. This hyper-verisimilitude removes even the camera as the creator of the work, ostensibly rendered out of existence by the perfection of its image [7].

Concomitant with these advances is the increase in the quantity of images made available by digital technology. Not only do the best arrive on the Internet, but pretty much everything else, so that we wade through a sea of images at every turn. While photographs enter the network within a particular context they do not remain there in perpetuity, and can soon break free to circulate and appear in multiple sites and locations. The plurality and mobility of the digital photograph leads to a dissociation of the image from its context in time and place and a peculiar sense of authorlessness, where even the fact that we are looking at an image made by a camera pointed by a human can escape our perception.

The foregoing commentary sets out the context in which the research project In Pursuit of Error [8] (hereafter referred to as IPE) emerged and seeks to challenge. The project collects photographic errors from artists, photographers, and the general public in order to form an archive of the error in contemporary practice. Contributors are asked to include a statement that explains how their photograph occurred and why they feel it is an error. Through analysis of the images and narratives, the project reveals how artists value the accident as a point of discovery and development and engage in playing with and against the camera in the creation of 'deliberate' errors. The project aims to question some of the assumptions about representation and truth which beset photography as a practice, and to challenge the assumption that technological developments manifest objectively rather than because of cultural and ideological assumptions about what is correct or good. Thus the project attempts to destabilize the hierarchy that attaches to the relationship between camera and photographer (with one or the other 'in control' at any given time) in favor of exposing the horizontality of this relationship [9, p. 283]. The project uses the error to explore a revitalized concept of photography as an embodied, performative practice, in which the bodies of photographer and camera play a part.

\subsection{Camera Acts and Human Acts}

In order to consider how humans and cameras interact in the creation of a photograph, it is necessary to think through the processes that bring a photograph into being. Despite common assumptions about time in relation to photography-the split second 'capture' - making a photograph has a number of stages, from the first desire to take a photo, to preparing or starting the camera, to focusing or selecting the composition through viewfinder or on screen and only then the taking of the photo. In 
this extended time period both camera and photographer perform a number of acts, the photographer her gestures, the camera ordered by the principles of its program. Within these parameters correct images are to be had; however, flouting the rules of either program or gesture tend to produce unexpected or unintended results.

It is useful to consider the practice of taking a photograph as operating on a continuum of control between agency and automatism. While one might assume that 'agency' pertains solely to the photographer and 'automatism' to the camera, in practice these roles are interchangeable [10]. Automatic gestures-holding the camera steady, or straight, or automatic programs such as auto-focus or aperture control create more or less expected results according to the scene presented. Agency plays its part in the decisions or mistakes of the photographer but the camera's agency can also intrude on the photographer's intentions, resulting in surprising and unexpected images.

The photographic errors submitted to IPE cover a range of accidental or unintended images and 'deliberate' errors in which a willful action has been made to achieve an unpredictable result. In many cases it is near impossible to identify from the resulting error image which party was the instigator, without the photographer's identifying narrative in support (Fig. 9.1). While the visual distinction between the two types of errors is not necessarily apparent, they create a useful distinction which permits

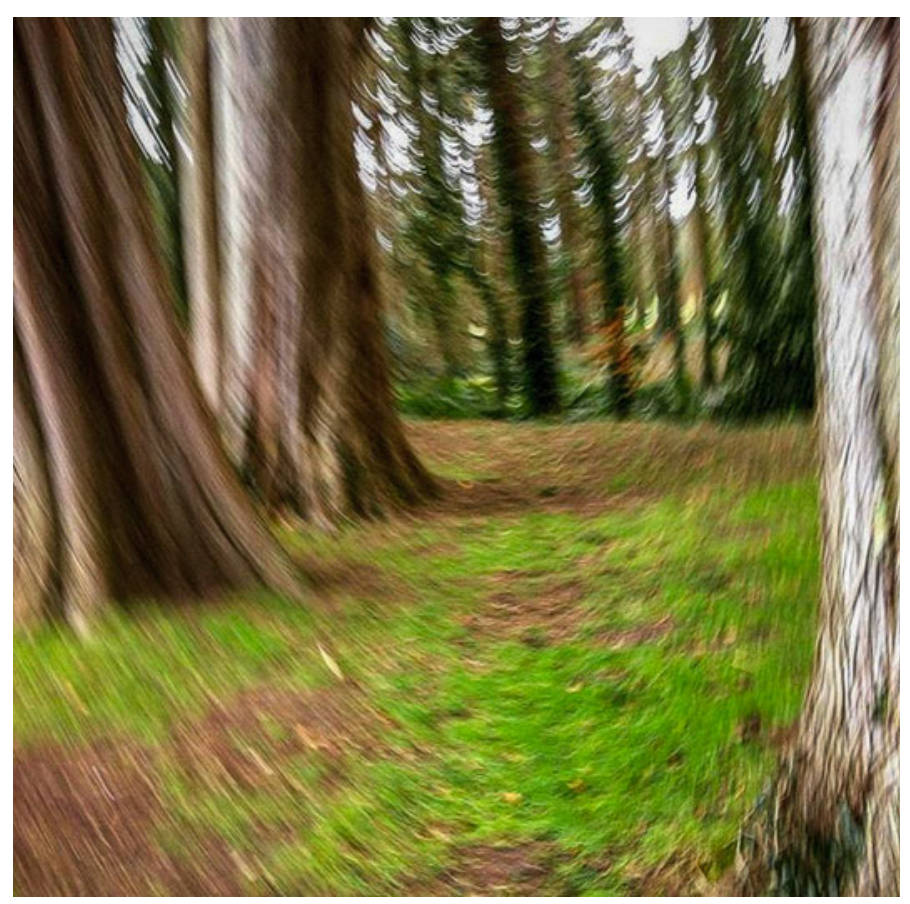

Fig. 9.1 Image copyright $\odot$ Gail Griggs and reproduced by permission. Source: In Pursuit of Error [8] 
a focus of attention on either actor in the event of photographing - the camera or the photographer. The narratives that accompany the contributions to IPE reveal the push and pull between the photographer's vision and the camera's vision, and where the presence of the camera as agent in the creation of the image is made apparent.

\subsection{Agents and Networks}

To consider the camera as an agent, or an accomplice, in the act of photographing is to reconsider the role of the technology and our relationship with it-as user, as master, or as collaborator. Actor-Network Theory (ANT) creates a framework through which to reconsider this relationship, based upon acknowledgement of the "symmetry of humans and non-humans" [10, p. 642] in networks. Networks are formed of social practices and technical processes as well as human and non-human 'actants' and vary in scale. A network could be as small as the photographer and her camera, or can be broadened out to consider the camera's developer and manufacturer, the photographer's educators and peers, and the social and cultural context of photography practice. ANT emerged from the sociology of science and technology as a means to critique assumptions about the development of technology in societies, pointing out relationships of power and questioning deterministic models of scientific development which present such development as rational and autonomous. ANT's non-hierarchical emphasis on 'actants' erodes distinctions between human and non-human actors and creates opportunities to consider how technology not only 'speaks back' to us through our interactions with it, but also how technology governs and en-frames our performance with it [11].

In the context of Actor-Network Theory the error sheds new light on the relationship between camera and photographer. Many of the accidental errors that are presented to IPE occur due to the mismatch between the photographer's intentions and the camera's program, or more strictly speaking, the settings that have been selected on the camera. Most modern cameras permit the pre-selection of aperture and shutter speed to account for the proximity of the subject and light conditions. Errors occur when the conditions fail to match the camera's settings, or vice versa (Fig. 9.2). In this case the situation being photographed becomes another actant in the network, a set of conditions which intrudes upon and affects the outcome.

In the surprise that is created by these unexpected images the photographer is made aware of their reduced role in the event of photographing, instead giving over the task of picturing to the camera. This mismatch between program and situation, 'translated' by the camera, presents a wholly new way of perceiving, a form of camera vision which reminds us of the subjectivity of perception per se. While gross mismatches between program and conditions create image-worlds far beyond our imagination, small collisions between settings and situation create spikes of maladjustment in the image through which the presence of the camera as an observing body can be detected. The accidental error unequivocally brings the camera's presence to the fore (Fig. 9.3). These images do not allow us to look 'through' or 'past' the photograph 
Fig. 9.2 Image copyright $(\odot$ Ian Wright and reproduced by permission. Source: In Pursuit of Error [8]

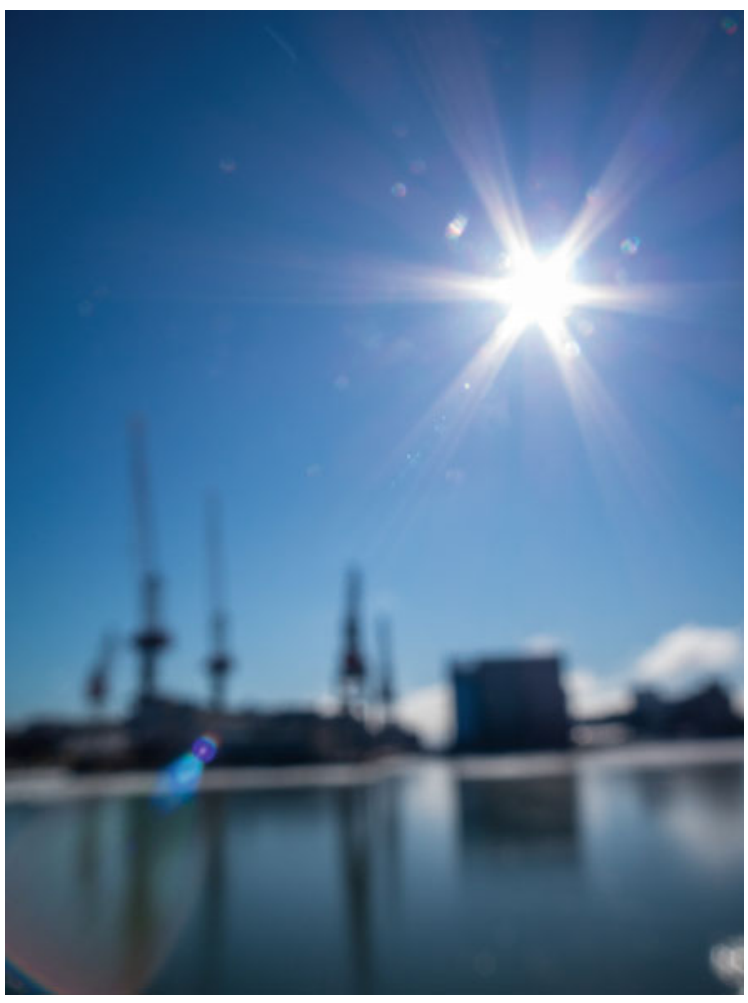

as medium, instead, through its faults, the 'photographicness' of the image is made glaringly apparent. The accident is therefore a valuable reminder of the technological mediation which the camera brings to the act of photography, a subjective vision which is easily overlooked in conventional photographs.

Photography's connection to the principles of objectivity and neutrality, which are socially and culturally grounded, have accustomed us to treat the camera as a perfect imaging-machine. The accident, revealing the fallibility of this conceit, reminds us that while automation exists, it is not a panacea which will offer unblemished facsimiles of the world every time. The accident demonstrates the camera's vision as subjectively driven rather than an objective fact and foregrounds the extent and limitations of the technology that lies behind that vision. These types of errors also reveal the role of the photographer in choosing the most appropriate functions to create an image, and thus the interdependence of camera and photographer in the event of photographing.

Not all accidents are the result of camera malfunction or wrong settings. Accidental images also arise through physical mishap (falling, shaking) or something inserting itself between the lens and the scene at the moment of capture. These intrusions into the photographic event evidence the wider field of actants in the Network of photographing. While the camera/photographer nexus would appear to be 


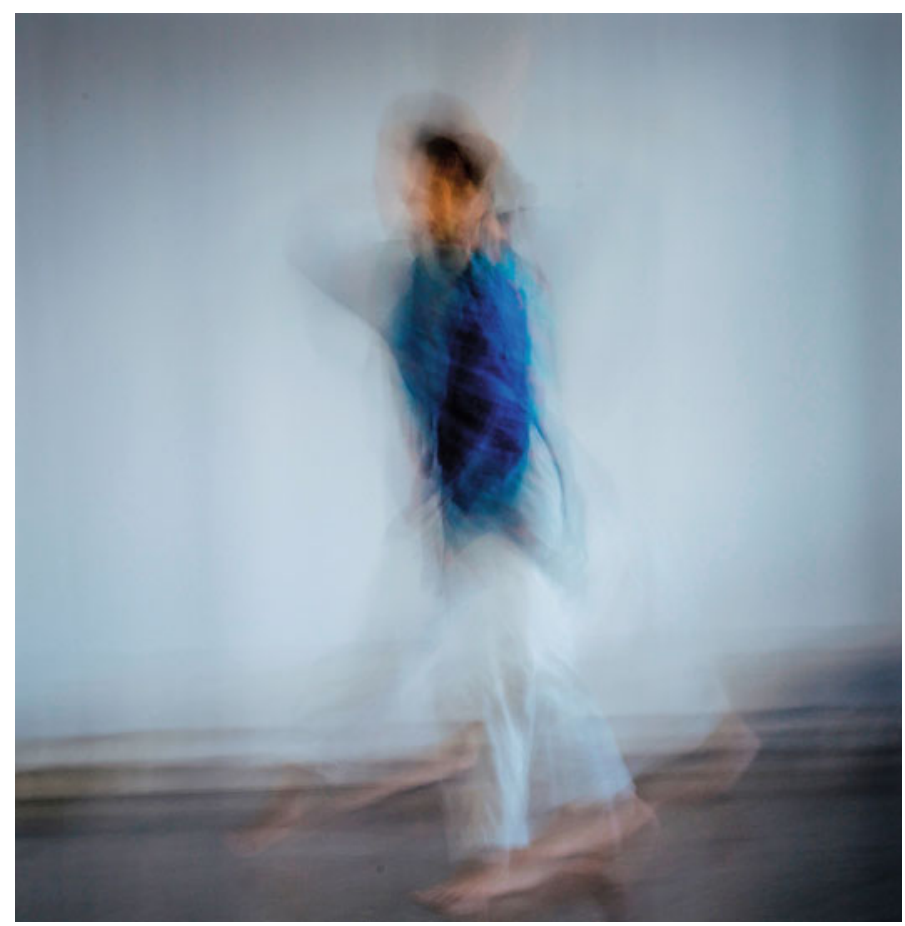

Fig. 9.3 Image copyright $(C$ Catalina Codreanu and reproduced by permission. Source: In Pursuit of Error [8]

the sole locus of creation, the error reveals the multiple secondary elements: light, time, subject, background; the numerous observable and unobservable phenomena that can impinge on the image. The accidental error reveals the momentariness of the photograph, its situatedness in time and space and its fixed location and viewpoint. Conventional photography practices strive to present a scene which is as far as possible universal, a witness to an event rather than a participant, working at an observing distance which lends the scene a sense of objectivity and 'truth'. This photographic stance, as common in newspaper reportage as holiday snaps, relies on a detachment between the photographer/camera and the scene, either through pose or 'stilling' of the action ('smile!') or through remaining on the periphery. By contrast, the accidental error provides incontrovertible evidence of the embeddedness of the camera/photographer within the flow of space and time through which pours all that is normally left out of the photograph (Fig. 9.4). Through happenstance the error records these fleeting intrusions and rapid changes that make up our customary lived experience, fixing for a second the swirl of life that we are part of.

Accidental errors create opportunities to observe the camera at work in the creation of the image, in many cases an overlooked or taken for granted act. For Borda [12] the physical materiality of the camera is in danger of being left behind in an increasingly 


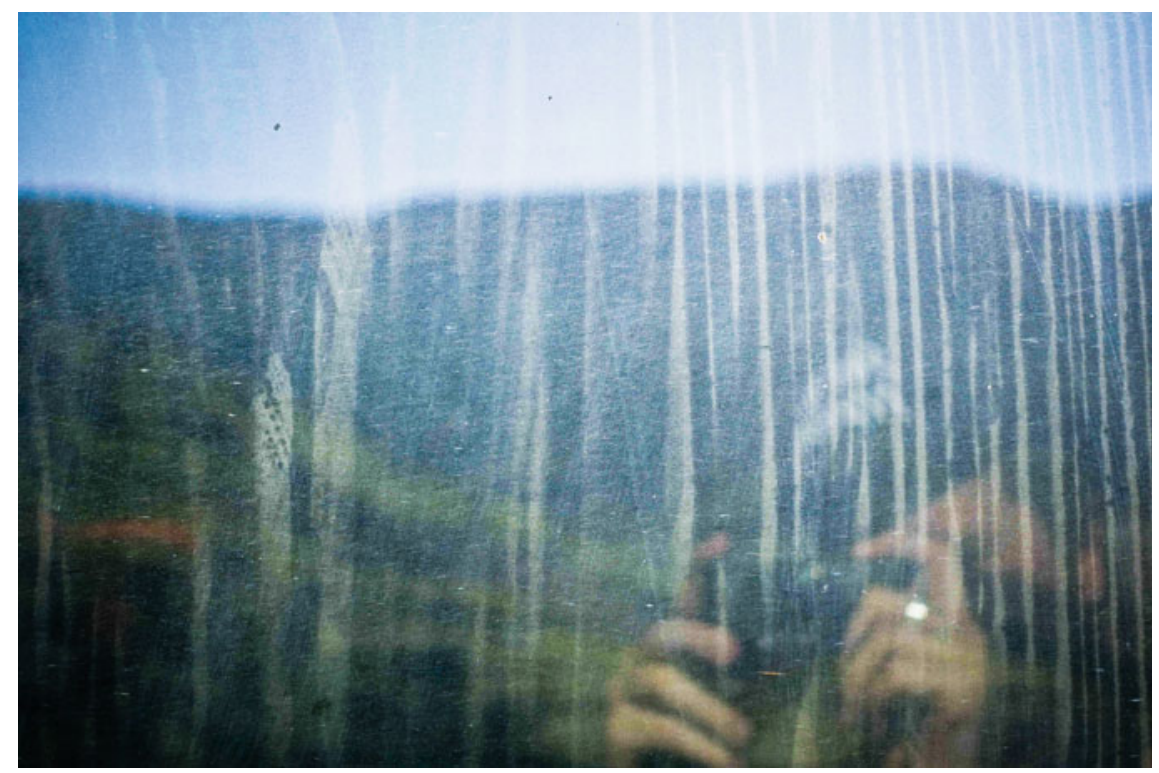

Fig. 9.4 Image copyright $(\odot$ Brett Chapman and reproduced by permission. Source: In Pursuit of Error [8]

digitized, automated picturing world which makes the camera "a missing entity that we no longer perceive." [12, p. 179]. The physicality of the camera has been greatly diminished by recent developments such as mirror-less technology which removes weight and mechanical action and in mobile phones which integrate the camera as a part of a hand-held computer. If the 'objectness' of the camera is somewhat at risk from these developments, the accidental error reasserts it as a form of what Jane Bennett would term 'thing power': the agency of material objects to act and impact other materials, thereby producing effects in the world [13]. The thing-power of the camera is writ large in the accidental error, where the photographer is left wondering what might have occurred, standing outside the making of the photograph, receiving the image as a fait accompli. For Bennett, acknowledging 'thing power' entails recognizing what she terms its 'out-side', that which we know to exist but can only indirectly perceive [13]. The camera exercises its 'thing power' through the hidden process of bringing the photograph into being. We know the theory but the actuality of the process is held in abeyance, because the chemistry or the program is closed to our perception [2]. This hiatus is where the camera exerts its agency and where for a few moments the balance of power between photographer and camera is tipped in the camera's favor. This gap in our knowledge should remind us that we are not necessarily masters of our technology, but rather informed collaborators, operating on a certain amount of knowledge and an element of trust. 


\subsection{From Tool to Plaything}

The hiatus that occurs in the moment of translation from event to image is an aspect of all photography, correct images, and mistakes alike. This loss of control, however momentary, seeps into the practice of photography and informs the relationship between camera and photographer which transpires to be a balance between knowing and not-knowing, of control and loss of control. Thus, photographs could be organized on a continuum from those made with complete knowledge and command, and those produced as a result of a complete loss of control and ignorance. The error could be said to occupy the middle to latter part of the continuum and thus express not a simple binary distinction between 'correct' or 'faulty' images but rather an elongated space of practice in which minor to major mistakes or maladjustments, made by either party, impact upon the eventual photograph.

Control could be considered a cornerstone of camera development through technological enhancements in efficiency and ease of use. The vagaries of the photographic process are brought under control by innovations such as cassette film, the digital sensor or 'Auto' pre-sets. The control is purportedly for the photographer, who can now manage the process more readily, but in fact the control, particularly in relation to programmable functions on modern digital cameras, is situated firmly with the camera, or perhaps we should say the camera's manufacturer. Elements of control can also be exercised through a culture of photographic practice; the way in which one should hold the camera or operate the shutter was often of particular concern in manuals for new style cameras where the photographer's performance with the new device was as much under scrutiny as the camera. Flusser points out that both 'apparatuses' (as he terms cameras) and photographers are subject to the control of the Program: the systems which define and limit the practice of photography [2]. Flusser is rather negative about the possibility of photographers escaping the limitations of the Program but he does recognize the agency of the individual in testing or subverting the expectations of picturing. For Flusser, the increasing functional capabilities of the camera, its automation of the photographic process, have liberated the photographer from the 'work' of making pictures. Now there is nothing else to do but play [2, p. 29].

Playing with the camera to undermine expected or 'correct' image making can be seen in the 'deliberate' errors, or rather, photographic subversions submitted to IPE. These images are often accompanied by a narrative that suggests the photographer's moment of performance with the camera and their action in the moment that has brought the image into being. Subversions often involve the photographer's physical movement, running counter to their expected role of stillness and stability-a human tripod which facilitates the camera's task. These movements can be complemented by a knowing destabilization of the camera functions-extensions of shutter speed to slow down the camera's capturing eye, forced de-focusing to render the scene into amorphous blobs of color (Fig. 9.5).

These actions could be characterized as playing against the camera, forcing it to see in a very different way through manipulation of the controls. Whether 


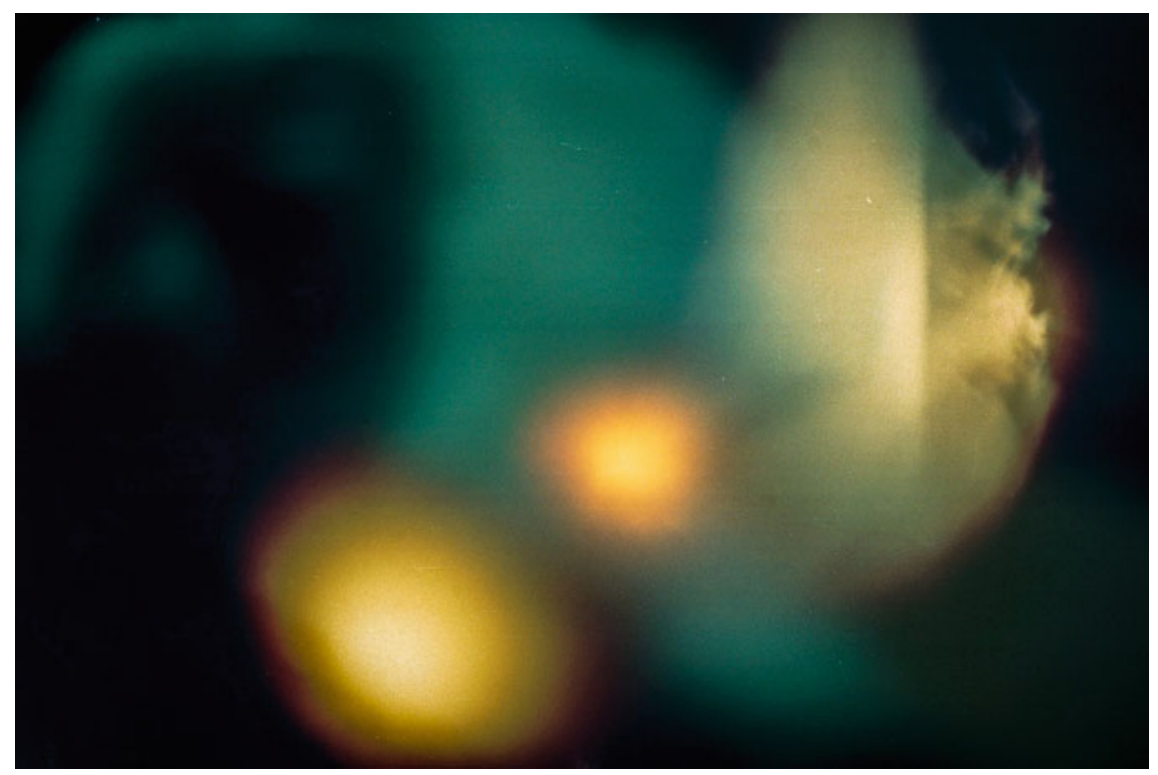

Fig. 9.5 Image copyright $(0)$ Melis Cantürk and reproduced by permission. Source: In Pursuit of Error [8]

through movement or manipulation the subversion foregrounds the photographer as an embodied presence in the act of making photographs in a similar way that the accident reveals the camera's subjective viewpoint. The subversion interposes the photographer between the camera and the scene as much as if a stray thumb or forefinger were to appear in the frame-we are reminded that the camera is not simply alone and recording by itself as an objective observer. The subversion reminds us that there is a photographer who is pointing, choosing, moving or otherwise governing the activity that precedes the image.

These subversions become traces of performance in time, capturing a certain configuration of events that involve camera, photographer, and situation. One may well ask why photographers feel compelled to intervene in the process of photographing in this way. Certainly, the desire to do so is evident throughout the history of photography, notably expressed as a principle of the Provoke photography movement in Japan in the 1950s in their mantra of 'are bure boke'_- "grainy, blurry, out of focus" [14]. Practices which test the conventions of photography can be seen in the work of practitioners as diverse as Meatyard [15] and Barth [16] whose out of focus experiments suggest alternative ways of using the camera as a subjective and sensory mode of expression rather than an objective recording device. The deliberate error is therefore more than just play; it is an urgent reassertion of creativity in the act of photography, one that seems to be increasingly marginalized by technological advancement which attempts to drive the unreliability of the human presence further away from the photographic act. 
Deliberate errors may also be motivated by a desire to not produce photographs. One of the main issues in photography theory has been the 'reality effect' of the photograph [17], that is to say that the photograph is not so much a created image as a recording, a document of what was in front of the lens at any given time. The reality effect is what lends photographs their 'transparency' [18], our tendency to look through photographs as material media to focus solely on what is depicted within the frame. The emphasis on transparency is what drives technological development, a quest to create a photograph which will be as real as being there, a pure and unadulterated 'slice of time'. As technological development makes this more possible it is little wonder that photographers expand their thoughts into new territory and tend to explore the different forms of picture-making that the camera may afford.

Elements of Francois Laruelle's thesis [19] can be brought to bear on the deliberate error in this regard. Laruelle attempts to disentangle photography from its grounding in doubling or copying by pointing out the philosophical distinction between the object in the world and its photographed representation [19]. Perceiving the object and its photographed form as the same is a fallacy that over-prioritizes the world and the objects in it, without considering the distinct ontology presented by the photographic image. For Laruelle claiming the non-identity of the photograph releases it from the dualities of the 'reality effect' and permits it to be a related but non-identical depiction of objects in the world. Set free in this way the photograph is no longer the poor handmaiden of reality but another presentation of reality which is distinct and unique to photography as a material practice $[19$, p. 55].

The potential of the photograph as a unique form of envisioning is suggested by Laruelle's commentary, and speaks of the practical understanding of photography as a transformative art. While embedded in an examination of the photograph as a philosophical problem, Laruelle's commentary expands photography from a simplified and primarily technological act toward an understanding of the central importance of the photographer/artist who, through the "spontaneous philosophy" of practice can reveal the "very essence of photography" [19, p. 85]. The photographers exploring the photographic subversion reveal the ambiguity at the heart of photography by making images that run counter to the principle of representation.

The creation of photographic subversions often centers upon an expansion of some kind: extended physical movement or elongated shutter times, which result in strange depictions of seemingly other-worldly phenomena-traces, trails, lines, and blurs which suggest but do not signify; the original scene lost behind a veil of color and movement (Fig. 9.6). These images present a divergent concept of photography which does not adhere to conventional understanding. They are often described as 'painterly', a direct response to the evidence of gesture and time that are inscribed on the image. They are in many respects, a form of 'non-photography' divested of the need to stand in for things in the world, instead presenting another reality which is lost to our normal human vision. For those who seek a deliberate error there is no need to make a photograph per se-we have machines that can do that for us. For the artist the liberation lies in the expanded capacity of the camera as a tool for seeing the world in radically revised ways. 
Fig. 9.6 Image copyright $($ ) Una $\mathrm{Li}$ and reproduced by permission. Source: In Pursuit of Error [8]

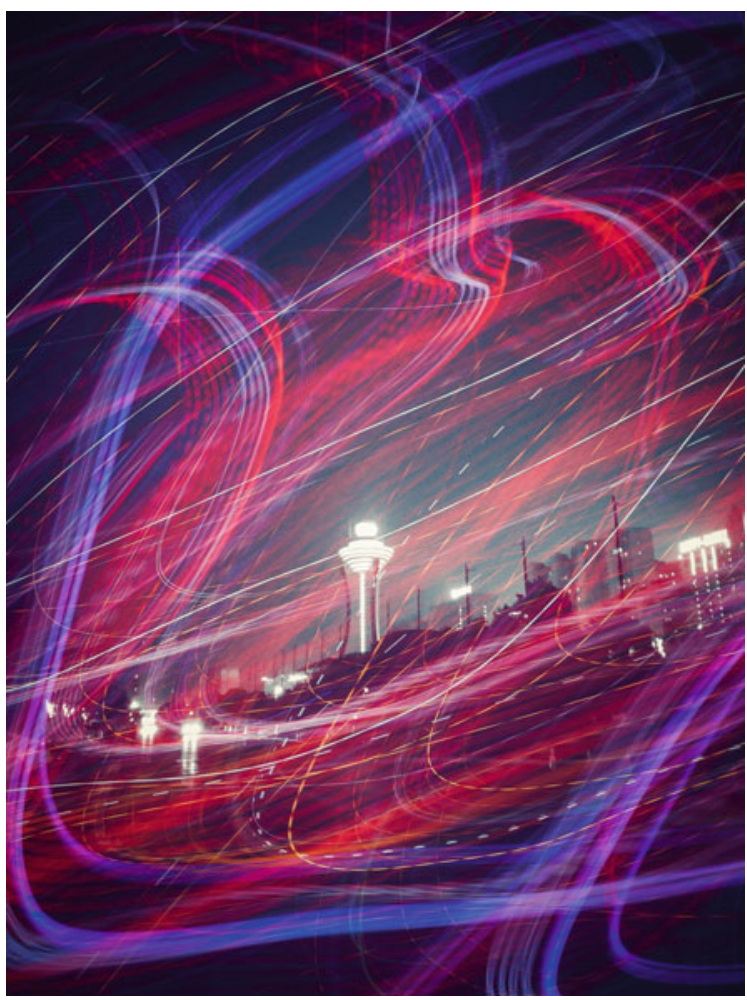

The satisfaction of the accident, and of being presented with the will of the material (or technology), is an intrinsic part of an artist's developmental method, their motivation to explore and progress. 'Not-knowing' drives practice forward [20].

In photography, the subversion contrives opportunities for not-knowing and to be surprised by unexpected outcomes. While a certain set of actions are put in motion it is not the case that the photographer can know how and to what extent they will affect the resulting image. The photographer may know that certain operations or actions are likely to produce one type of result, but she cannot predict the image outcome as when making a photograph in the conventional way. The subversion lies further toward the knowledge/control end of the continuum discussed at the start of this section, but it shares similarities with the accident in the gap that opens between intentions and outcome. This gap is made up of the present tense performance of photographing and the combination of photographer, camera, light, time, subject, and situation which coalesce in the resulting image. The error is an improvisation, always occurring in the immediate, unscripted moment and thus unknowable until that moment has passed. 
For photographers who engage in the deliberate subversion of photographic protocol the results of their transgression offer a similar level of satisfaction to encountering an accident, and can perhaps offer new avenues to explore in practice. The possibility of a different type of photography is offered by the artists who tread this path.

\subsection{Tinkering with Technology}

The foregoing has shown how the error steps outside the conventions of photographic practice and reveals a more complex relationship between camera and photographer than that between an 'operator' and her 'apparatus'. Photographers often have personal relationships with their cameras, forming a kind of symbiosis between actants where one knows what a particular camera will do in a given situation. The photographer who engages in 'camera vision' connects to the photographic seeing that is a required element of making good pictures and yet as we have seen this can be disrupted by an unexpected event or knowingly cast aside. These apparent 'failures' could be seen as end points, or dead ends from which one must reverse or retreat, or they could be seen as opportunities presenting new phenomena which stimulate the imagination. Invariably the contributors to IPE couch their errors in this latter context, as intriguing surprises which stimulate their practice or their perception about what a photograph is or could be. The etymological root of 'error' lies in the notion of 'wandering', suggesting a deviation which is both intended and imbedded in not-knowing as a principle [21, p. 21]. To wander is to be open to the possibilities that arise in that act of wandering, be they positive or negative and might also imply a return to the conventional route at a later point. This conception of error encapsulates how photographers use the error in practice to lay claim to previously unexplored territory.

The territory occupied by the standards and procedures for 'correct' photography is vast and well developed. Since the very early inception of the medium, photographic practice has been intrinsically tied to rules on the right way to use the technology in order to produce good photographs. Some guidelines were initially necessitated by the complex and awkward materials and processes entailed in early photography, but rapid development of photographic substrates meant that processes soon became easier, quicker, and cheaper. Rules are not made by technology itself, but by the culture that surrounds and uses it. In the case of photography, the emphasis on progress and refinement exemplified through rapid technological development worked alongside a perceived need to regulate and define the limits of this emergent art form. Like all technologies, photography is deeply embedded in the social and political structures that helped formulate it as a popular and commercial entity and which continue to inform its development. In this network of influences, cultures of practice emerge.

The most pertinent distinction in the practice of photography is between the informed and the casual photographer, a distinction evident since the early days 
of the medium's history. The informed amateur photographer was an early adopter of new technology, in some cases contributing to the development of the medium through their experiments. Informed photographers created Photographic Societies where they could discuss and disseminate treatises on the art and science of photography, organizing its philosophical principles, its areas of concern, and its goals. The casual photographer emerged as the medium became simplified and democratized by the new easier to use substrates and cameras, culminating in the Kodak, which offered them a means to enter the realms of photography without the necessary skills and knowledge to create their pictures other than a willingness to point and shoot. The first fully-fledged 'movement' in photography, Pictorialism, emerged as a reaction to these new 'snapshooters' and to the automation and loss of human skill that the new developments suggested for the medium [22].

Thus the technological development of photography, driven by democratization and commerce, contributed to a fracturing of perceived elements of skill and knowledge that were in the guardianship of a cognoscenti. Kodak's invention forced the Pictorialists towards increasingly 'unphotographic' works - those that capitalized on the artist/photographer's hand to destabilize the verisimilitude of the image, turning it instead into proto-painting [22]. The casual photographers, freed from concern about the quality of their images by the fast and easy methods available to them, took more chances with their photos; and errors, mistakes, and mishaps became part of the photographic landscape [23]. As Hand points out, this entry of the banal mistake into the culture of photography practice challenged "the dominant discursive and material legitimacy of photography, especially in terms of skill and expertise." [24, p. 104].

Arguably, this distinction between informed/casual photographers is still recognizable today. The camera clubs of Britain are filled with those who pride themselves on their technical skills and expertise while casual photographers take selfies on their mobile phones. Photography's intrinsic link with technology makes this distinction, and the value placed on technical know-how, continually pertinent today.

The distinction between an informed and a casual photographer therefore lies in the amount of control they are able or willing to exert over the technology of photographing, which includes the camera, recording medium, and image production. The informed photographer, through her expertise, exercises control over the technology, mastering its principles to produce 'correct' photographs. The casual photographer is a receiver of the technology and perhaps somewhat subservient to it in that its expertise appears to outstrip his own. With less emphasis on control needed or desired by the casual photographer, technological development for this market expands with handy, time-saving innovations which bring the 'ideal' photograph within easy reach.

Thus, two paths of activity are suggested by cultures of practice in photography in which the level of control over the events of photographing favor one or other actant. As has already been demonstrated, the error appears to undermine this dichotomy and suggests a third way of working with photographic technology which is akin to Sherry Turkle's concept of 'soft mastery' [25, p. 56]. This concept emerged from a study carried out by Turkle and Papert on student programming styles [26] which revealed 
differences between those who took a logical, top-down approach to a programming problem, and those who preferred to work in an iterative, exploratory way with the computer. The distinction between 'planners' and 'bricoleurs' was expressed in a willingness, or otherwise, to engage with the computer as more than just a 'tool' but as a collaborator in the process of developing the program, exploring the nuances of its unique language and exploiting novel solutions to problems. Observation of different programming styles revealed that two approaches were at work; on the one hand there was a level of engagement with the process of programming as a problem which unfolded in time, and on the other, an approach which sought to previsualize all the aspects of the program before commencing work. This distinction in approach revealed a difference in the quality of the relationship between programmer and computer, which could be characterized for the bricoleur as a dialog and for the planner as a 'monologue' [26, p. 136]. The notion of conversation entails responding to the other party and perhaps adapting the flow of the conversation in accordance with those responses, while a monologue implies a silent audience. When errors occurred in the programming process, planners and bricoleurs differed markedly with how they dealt with this, either as failure or as opportunity:

For planners, mistakes are missteps; for bricoleurs they are the essence of a navigation by mid-course correction. For planners a program is an instrument for premeditated control; bricoleurs have goals, but set out realize them in the spirit of a collaborative venture with the machine [26, p. 136].

Turkle's concept of 'soft mastery' expresses a position of informed knowledge about the goal or the object to be realized, but an epistemologically different way of making the journey. Soft mastery relies on a form of negotiation with the materiality of the object in question, be it a computer or a camera, a programming language or abstract problem. Turkle adapts the concept of the bricoleur from Claude LeviStrauss's descriptions of knowledge creation in primitive societies, what he terms a "science of the concrete" [26, p. 135]. To be concrete is to be present, tangible, handleable, not abstract and conceptual. This notion of the presence of objects as sets of things that can act and be acted upon is pertinent to a different way of understanding the camera, not as a tool to be mastered conceptually but as a thing to be worked with physically by testing it out and seeing what it will do.

This approach is readily witnessed in photographers who experiment with the camera and suggests a level of playfulness and willingness to 'see what happens' when using the technology. This kind of 'tinkering' with the parameters of photographing can be seen in many of the contributions to IPE. It's important to note that Turkle's concept of 'soft mastery' does not set out to simply reverse the dichotomy between logic and practice, control and experimentation, but to expose the hierarchy that would assume that logic and control were the only or best ways of engaging with technology. The type of knowledge created through negotiation, through practice, reveals alternative epistemologies that run counter to the status quo [26].

A good example of a bricoleur approach to photography can be found in the work of Julia Margaret Cameron. Cameron was an early pioneer of photography as an art form, creating striking portraits of friends and family in scenarios influenced by 
literature and painting. A particular feature of Cameron's photography is a deliberate softness of focus. In her extensive writings she notes how this particular feature of her work arose in practice, and led her to adopt it as a signature style:

\footnotetext{
I believe...that my first successes in my out-of-focus pictures were a fluke. That is to say, that when focusing and coming to something which, to my eye, was very beautiful, I stopped there instead of screwing on the lens to the more definite focus which all other photographers insist upon [27, p. 77].
}

It is pertinent that while having complete command of the camera as a piece of technology, Cameron instead chose to pursue a deliberate 'mistake' or subversion of the accepted protocols for photographing in order to compose an image which was closer to her intentions. Cameron's informed but embodied interaction with the camera was deemed 'sloppy' and incompetent by those who had set themselves as gatekeepers for the craft, a regulatory status quo which set the rules by which photographs were to be made [28]. The fact that Cameron refers to them as her 'out of focus pictures' is perhaps a significant internalization of this criticism in a nascent world of cultures of practice which were being formed and entrenched.

What Cameron's early flouting of the 'rules' of photography suggests is a connection between photographer and camera in which the photographer becomes aware of the frailties and limitations of the technology, and the creative potential that these peripheries afford. This awareness stems from a willingness to get inside the camera in some way, to envisage its 'camera seeing' as a unique form of agency which has a contribution to make to the final image. In their study Turkle and Papert observe this type of investment into inanimate objects as a form of "reasoning from within" [26, p. 144] which is embodied rather than abstract. Reasoning from within requires a proximal relationship with the objects that we use, rather than a distance which views them as purely functional tools. To acknowledge the camera's presence in the making of a photograph is to acknowledge it as a collaborator and changes our relationship with it.

Entry to this new way of thinking about technology might be as simple as the first time we encounter something that has gone wrong. Bill Brown observes that we rarely notice the objects that surround us when they are working correctly. In this state they become invisible; we almost see past them, or through them. However, when something goes awry we are forced to reckon with the object as a different order of 'thing', an agent that has impacted on us in an unforeseen way. The breakdown or interruption in the flow of expected behavior changes our relationship with the object and redefines it [29].

Error, mistakes, tinkering, and subversion are therefore vital and important aspects of photography as a creative practice. They create gaps in the armor of accepted practice, chinks of light where other possibilities for photography can emerge and be explored. The epistemological pluralism espoused by Turkle and Papert [26] exposes a new way of relating to photographic technology; as an embodied, proximal relationship between two agents, two actors who negotiate in the creation of a photograph. 


\subsection{Conclusion: Being in Two Minds}

The foregoing has suggested some alternative ways to consider the relationship between camera and photographer, and how cultures of practice emerge which reinforce oppositional hierarchies between users and their tools. The power of the accident or the subversion to undermine some of the dominant metaphors that adhere to photography-facsimile, accuracy, perfection-exposes a counter culture of photographic practice which has been explored and exploited by practitioners since the early days of the medium.

To adopt the embodied and proximal methodology of the bricoleur is to be open to a sense of discovery and unfolding in the creative process, a form of confident 'not-knowing' from which to begin.

Vilem Flusser observes that "The act of photography is that of 'phenomenological doubt'." [2 p. 38]. For Flusser doubt in photography is expressed through the potential multiple viewpoints enabled by the camera and the aspects of the photographic process which are invisible to us. Doubt is woven into the elements of the photographic event in which multiple actants converge at a moment in time and in the hiatus of control and decision-making that is characterized by the accident or the subversion.

The etymology of doubt in 'doubling' in the sense of having to choose between two things [30] offers another way of interpreting the not-knowing that we experience in the event of photographing. To doubt is to be in two minds or to assume two positions. The error exposes this doubling as it occurs in practice, as a dance between two epistemological positions: to know and not know, to be in control and to relinquish control, and as a dance between two actors: camera and photographer. To be in two minds is to enter a position which permits a relational flow between machine vision and the embodied photographer, between presence and program, in which the merging of both exceeds the power of either.

\section{References}

1. Deleuze, G.: Bergsonism. Translated by H. Tomlinson and B. Habberjam. Zone, New York (1988)

2. Flusser, V.: Towards a Philosophy of Photography. Translated by A. Mathews. Reaktion, London (2000)

3. Parker Willis, N., Porter, T.O. (eds.): The Pencil of Nature. A New Discovery. The Corsair. 1 No. 5 (Saturday April 13): 70-72. Reprinted in Photographic Theory: An Historical Anthology. In: Hershberger, A. (ed.) Wiley-Blackwell, Oxford, pp. 44-47 (2014)

4. Da Vinci, L. C.: The Function of the Eye. As Explained by the Camera Obscura in Photographic Theory: An Historical Anthology. In: Hershberger, A. (ed.) Wiley-Blackwell, Oxford, pp. 1718 (2014)

5. Petchesky, R.P.: Fetal Images: The power of visual culture in the politics of reproduction. Fem. Stud. 13(2), 263-292 (Summer, 1987)

6. Pritchard, M.: A History of Photography in 50 Cameras. Bloomsbury, London (2016) 
7. Bate, D.: The digital condition of photography: cameras, computers and display. In: Lister, M. (ed.) The Photographic Image in Digital Culture, 2nd edn. Routledge, London, pp. 77-94 (2013)

8. Piper-Wright, T.: In Pursuit of Error [Online]. www.inpursuitoferror.co.uk

9. Frow, J.: A pebble, a camera, a man who turns into a telegraph pole. Crit. Inq. 28(1), 270-285 (Autumn 2001)

10. Feenberg, A.: A critical theory of technology. In: Felt, U., Fouché, Clark, R., Miller, A., Smith-Doerr, L. (eds) The Handbook of Science and Technology Studies, 4th edn. MIT Press, Cambridge Mass, Cambridge, MA, pp. 635-663 (2017)

11. Latour, B.: On actor-network theory: a few clarifications. Soziale Welt Jahrg H 4, 369-381 (1996)

12. Borda, S.G.: Locating the camera in a digital time. Photographies 11(2-3), 177-192 (2018)

13. Bennett, J.: Vibrant Matter, A Political Ecology of Things. Duke University Press, Durham, $\mathrm{NC}(2010)$

14. Chong, D.: Tokyo 1955-1970 A New Avant Garde. The Museum of Modern Art, New York (2012)

15. Meatyard, R.E.: Learning to see 'No-Focus' (2011) [Online]. https://www.americansuburbx. com/2013/01/ralph-eugene-meatyard-reframing-photography-theory-and-practice-excerpt2011.html

16. Barth, U.: Artist's website [Online]. http://utabarth.net/work/untitled-1998-99/

17. Barthes, R.: In: Barthes Reader, A., Sontag, S. (ed.) The Photographic Message [1961]. Hill and Wang, New York, pp. 194-210 (1982)

18. Walton, K.: Transparent pictures: on the nature of photographic realism. Crit. Inq. 11(2), 246277 (1984)

19. Laruelle, F.: The Concept of Non-Photography. Translated by R. Mackay. Sequence Press, New York (2011)

20. Barthelme, D.: In: Herzinger, K. (ed) Not Knowing in Not Knowing: The Essays and Interviews of Donald Barthelme. Random House, New York (1997)

21. Nunes, M. (ed): Error: Glitch, Noise and Jam in New Media Cultures. Continuum Books, London (2011)

22. Warner Marien, M.: Photography: A Cultural History, 4th edn. Laurence King, London (2014)

23. Hirsch, R.: Seizing the Light: A History of Photography. McGraw-Hill, Toronto (2000)

24. Hand, M.: Ubiquitous Photography. Polity Press, Cambridge (2012)

25. Turkle, S.: Life on the Screen: Identity in the Age of the Internet. Simon \& Schuster, New York (1995)

26. Turkle, S., Papert, S.: Epistemological pluralism: styles and voices within the computer culture. Signs 16(1), 128-157 (1990)

27. Cameron, J.M.: Annals of My Glass House. First Published in Photo Beacon (Chicago) 2 (1890), pp. 157-160. In: Hershberger, A. (ed.) Photographic Theory: An Historical Anthology. Wiley-Blackwell, Oxford, pp. 76-79 (Reprinted) (2014)

28. Weaver, M.: Julia Margaret Cameron 1815-1879. Little Brown Company, Boston, MA (1984)

29. Brown, B.: Thing theory. Crit. Inq. 28(1), 1-22 (Autumn, 2001)

30. Klein, E.: A Comprehensive Etymological Dictionary of the English Language. Elsevier, London (1969) 
Open Access This chapter is licensed under the terms of the Creative Commons Attribution 4.0 International License (http://creativecommons.org/licenses/by/4.0/), which permits use, sharing, adaptation, distribution and reproduction in any medium or format, as long as you give appropriate credit to the original author(s) and the source, provide a link to the Creative Commons license and indicate if changes were made.

The images or other third party material in this chapter are included in the chapter's Creative Commons license, unless indicated otherwise in a credit line to the material. If material is not included in the chapter's Creative Commons license and your intended use is not permitted by statutory regulation or exceeds the permitted use, you will need to obtain permission directly from the copyright holder.

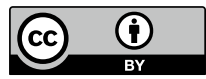

